\title{
Diseño de políticas de sostenibilidad y prácticas ciudadanas. El caso del comercio y las agendas 21 locales
}

\section{Josep Espluga Trenc}

Universitat Autònoma de Barcelona. Departament de Sociologia

joseplluis.espluga@uab.cat

\section{Àlex Boso}

Universidad de la Frontera. Núcleo Científico Tecnológico en Ciencias Sociales y Humanidades alex.boso@ufrontera.cl

\section{Ana Prades}

Centro de Investigaciones Energéticas, Medioambientales y Tecnológicas.

Centro de Investigación Socio-Técnica (CIEMAT-CISOT)

ana.prades@ciemat.es

\section{Resumen}

En el presente artículo, se analiza, en primer lugar, la distancia entre lo que la gente dice y lo que la gente hace en relación con la sostenibilidad en su vida cotidiana; en segundo lugar, se intenta conectar este conocimiento con el diseño y la elaboración de políticas de sostenibilidad. Para ello, se ha escogido el caso de una muestra de comerciantes de la ciudad de Barcelona y su relación con las políticas que intentan promover la sostenibilidad en la ciudad (la Agenda 21 Local). Se ha utilizado una metodología híbrida denominada STAVE, que combina las técnicas del grupo de discusión con métodos iterativos y reflexivos por medio de una orientación de investigación-acción. Los resultados permiten identificar las predisposiciones de los comerciantes hacia la sostenibilidad, así como detectar en qué medida sus discursos son coherentes con sus prácticas. Además, los resultados muestran hasta qué punto las políticas municipales de la Agenda 21 de Barcelona pueden encajar en los contextos cotidianos del comercio local para promover avances hacia la sostenibilidad en el sector.

Palabras clave: sostenibilidad; Agenda 21; comercio local; políticas ambientales; percepción social; método STAVE. 
Abstract. The design of policies on sustainability and citizen practices: The case of local shopkeepers and the Agenda 21

This article first analyses the distance between what people say and what people do in relation to sustainability in their daily lives; and, secondly, it tries to connect this knowledge to the design and development of sustainability policies. We have chosen a case study based on a sample of shopkeepers in the city of Barcelona, and their relationships with policies seeking to promote sustainability in the city (LA21). We have used an hybrid methodology called STAVE that combines the techniques of reconvened focus group and thoughtful iterative methods with an action research orientation. The results allow us to identify shopkeeper's predispositions towards sustainability, and to identify to what extent their discourses are consistent (or not) with their practices. Furthermore, the results show how municipal policies of the Local Agenda 21 can fit into everyday contexts of shopkeepers to promote progress towards sustainability in the commercial sector.

Keywords: sustainability; Agenda 21; local trade sector; environmental policies; social perception; STAVE methodology.

\section{Sumario}

1. Introducción: La A21 como programa político para la sostenibilidad

2. El método STAVE

3. El estudio de caso: ¿Cómo puede contribuir la A21L de Barcelona a conseguir que el pequeño comercio local avance hacia la sostenibilidad ambiental?
4. Resultados

5. Conclusiones

Agradecimientos

Referencias bibliográficas

\section{Introducción: La A21 como programa político para la sostenibilidad}

\subsection{La A21L de Barcelona}

En la Conferencia de las Naciones Unidas sobre el Medio Ambiente y el Desarrollo (conocida también como Cumbre de la Tierra), celebrada en Río de Janeiro en 1992, se acordó el plan de trabajo denominado Agenda 21 para avanzar hacia el «desarrollo sostenible». El documento de la Agenda 21 recapitulaba todas las problemáticas relacionadas con el medio ambiente y el desarrollo, además, en su capítulo 28 , proponía la conveniencia de contextualizar los objetivos generales del programa en las comunidades locales, para lo que se sugería que elaboraran un plan de actuación concreto: la Agenda 21 Local (A21L). Durante los años siguientes, miles de municipios de todo el mundo impulsaron sus propias A21L.

El Ayuntamiento de Barcelona auspició la constitución, en 1998, del llamado Consejo Municipal de Medio Ambiente i Sostenibilidad, una mesa participativa formada por representantes de asociaciones locales, universidades, empresas e instituciones públicas que, durante tres años, realizó trabajos para definir los principales retos ambientales de la ciudad y proponer los objetivos 
adecuados para avanzar hacia una ciudad más sostenible. Este proceso fue objeto de información y debate en amplios sectores ciudadanos durante el año 2001 y culminó con el establecimiento del llamado Compromiso ciudadano por la sostenibilidad, una declaración de intenciones que permitió definir 10 objetivos generales ${ }^{1}$.

A su vez, para cada objetivo, se establecieron 10 indicadores (100 indicadores en total) que permitirían medir y evaluar los presuntos avances de la ciudad hacia la sostenibilidad.

Para promover los objetivos de la A21L, el Ayuntamiento invitó a todas las entidades, asociaciones, empresas privadas e instituciones públicas del municipio a firmar voluntariamente el Compromiso ciudadano por la sostenibilidad. Esta política se denominó Acción 21 y supuso la formalización del compromiso de tal manera que se configuró una «red de firmantes» que ha tenido un importante protagonismo en la consecución de aquellos objetivos y en su seguimiento a lo largo del tiempo. El programa Acción 21 se planificó para un periodo de 10 años (2002-2012). Cada entidad firmante se comprometía a hacer un autodiagnóstico en cuanto a la sostenibilidad, a elegir uno o más objetivos sobre los que actuar y a elaborar un plan de acción que recogiera las medidas que se llevarían a cabo, el calendario y la asignación de responsabilidades internas para alcanzarlas. En 2011, las organizaciones firmantes del Compromiso ciudadano por la sostenibilidad eran 721, de las cuales 120 habían hecho público su plan de acción, además de los 283 centros educativos que lo desarrollaban en el marco del programa Agenda 21 Escolar durante el curso 2010-2011 (Ayuntamiento de Barcelona, 2011). En 2015, siguen registradas 751 empresas y entidades — de las que 346 son escuelas- que forman parte de la red.

En el año 2012, se realizó un nuevo proceso participativo para evaluar y renovar los objetivos del Compromiso ciudadano por la sostenibilidad, de manera que, a lo largo de varios meses, se realizaron reuniones temáticas y fórums virtuales que permitieron la elaboración y la aprobación de un nuevo programa. Así, desde 2012, la A21L promueve el programa Barcelona + Sostenible, que se articula alrededor de un nuevo plan de trabajo basado en 10 objetivos remodelados, relacionados con los 10 del programa anterior,

1. Los 10 objetivos del Compromiso ciudadano por la sostenibilidad que se adoptaron en 2002 fueron los siguientes (Ajuntament de Barcelona, 2002a: 7):

1. Proteger los espacios libres y la biodiversidad y ampliar las zonas verdes urbanas.

2. Defender la ciudad compacta y diversa, con un espacio público de calidad.

3. Mejorar la movilidad y hacer de la calle un entorno acogedor.

4. Alcanzar niveles óptimos de calidad ambiental y conseguir una ciudad saludable.

5. Preservar los recursos naturales y promover el uso de los renovables.

6. Reducir la producción de residuos y fomentar la cultura de la reutilización y el reciclaje.

7. Aumentar la cohesión social, fortaleciendo los mecanismos de equidad y participación.

8. Potenciar la actividad económica orientada hacia un desarrollo sostenible.

9. Progresar en la cultura de la sostenibilidad mediante la educación y la comunicación.

10. Reducir el impacto de la ciudad sobre el planeta y promover la cooperación internacional. 
pero redefinidos en función de la evolución social, económica y ambiental de la última década ${ }^{2}$. Además, para cada objetivo, se definieron 10 nuevos indicadores de seguimiento y evaluación.

\subsection{La Agenda 21 de Barcelona y el comercio local}

Entre el amplio conjunto de entidades, asociaciones y empresas firmantes del Compromiso ciudadano por la sostenibilidad de la ciudad de Barcelona, la presencia de comerciantes es muy escasa (solo 9 comercios aparecen registrados en la web de Acción 21), a pesar de que, entre los firmantes, se encuentra el llamado Consejo de Gremios de Comercio, Servicio y Turismo de Barcelona (CG), algo así como la patronal del sector. Según su propia descripción, «el CG es una entidad de representación empresarial y sectorial creada en 1980 para la defensa, representación y fomento de los intereses generales de los sectores del comercio, los servicios y del turismo de Barcelona y comarcas. Está integrado por 22 gremios y asociaciones sectoriales que agrupan cerca de 25.000 pequeñas y medianas empresas» (CG, 2010).

Desde el punto de vista de los responsables de la A21L de Barcelona, el comercio local podría desempeñar un papel clave en la mejora ambiental de la ciudad. De acuerdo con la Guía metodológica para avanzar hacia la sostenibilidad en Barcelona (Ajuntament de Barcelona, 2002b), editada por el propio Ayuntamiento:

El potencial de actuación del sector comercial es muy importante y abarca muchos ámbitos sociales y ambientales (energía, agua, residuos, comercio justo, divulgación, etc.), ya que por los más de 60.000 establecimientos que cada día abren sus puertas a la ciudad pasa buena parte de la población barcelonesa para hacer sus compras. Así pues, cualquier acción en la línea del desarrollo sostenible que se lleve a cabo en el ámbito del sector puede tener un impacto comunicativo de gran magnitud. (Ajuntament de Barcelona, 2002b: 34-35)

2. Los 10 objetivos del nuevo Compromiso ciudadano por la sostenibilidad 2012-2022 son los siguientes (Ajuntament de Barcelona, 2012: 9):

1. Biodiversidad: del verde urbano a la renaturalización de la ciudad.

2. Espacio público y movilidad: de la calle para circular a la calle para vivir.

3. Calidad ambiental y salud: de los estándares a la excelencia.

4. Ciudad eficiente, productiva y de emisiones cero: de la Barcelona tecnológica a la Barcelona inteligente.

5. Uso racional de los recursos: de la sociedad de consumo al consumo responsable.

6. Buen gobierno y responsabilidad social: de la intervención sectorial a la coordinación efectiva.

7. Bienestar de las personas: de la ciudad acogedora a la sociedad cohesionada.

8. Progreso y desarrollo: de la preocupación por la sostenibilidad a una economía que se fundamente en ella.

9. Educación y acción ciudadana: de la concienciación a la corresponsabilización con conocimiento de causa.

10. Resiliencia y responsabilidad planetaria: de la respuesta puntual a la acción global. 
Una encuesta del propio CG, realizada con el apoyo de la A21L de Barcelona muestra en qué punto se encuentra la sensibilidad y las prácticas ambientales de los pequeños y medianos comerciantes de la ciudad. La encuesta se realizó a 600 establecimientos comerciales (en 2010; con un error muestral del 4\% y un nivel de confianza del 95,5\%, p/q 50\%). Nos centramos aquí en los resultados de la encuesta para tres de los objetivos del Compromiso ciudadano por la sostenibilidad (los objetivos relativos a residuos, recursos naturales e impacto en el planeta), que, de acuerdo con las orientaciones del Ayuntamiento, serían los más susceptibles de motivar posibles acciones por parte del comercio local hacia la sostenibilidad (CG, 2010).

A modo de síntesis, los datos de esta encuesta muestran que la mayoría de los comerciantes dice seguir unas buenas prácticas de reducción de consumo de agua (el 70,3\%) y de energía (el 81,8\%), venden mayoritariamente productos respetuosos con el medio (el 68,2\%) e informan a sus clientes sobre diversos aspectos ambientales a través de las etiquetas (el 69,5\%) o comunicando la eficiencia energética de los electrodomésticos (el 88,9\%). Además, su práctica totalidad (más del 95\%) asegura seleccionar los residuos que genera y depositarlos en los lugares establecidos por el Ayuntamiento. Asimismo, la gran mayoría (el 77,2\%) dice que minimiza los envoltorios que da a sus clientes y les informa sobre la correcta gestión de los residuos electrónicos (el 69,2\%).

Sin embargo, al mismo tiempo que afirman todo lo anterior, la mayoría de ellos (el 63,2\%) dice ignorar la incidencia ambiental de los productos que vende. Y en cuanto a la selección de residuos, parece que solo el papel y el cartón son gestionados correctamente en una proporción elevada (el 87,8\%), porcentaje bastante más bajo en el caso de los plásticos (el 55,5\%) y de la materia orgánica (el 31,5\%), y mucho menor todavía en el resto: vidrio (el 18,0\%), aceites (el 10\%) y metales (el 5,3\%). Además, la mayoría de los comerciantes no tiene ninguna estrategia de reducción de residuos (el 76,2\%), no ofrece productos en envases retornables (el 66,4\%), ni bolsas de papel en lugar de las de plástico (el 61,0\%), ni explica a sus clientes qué hacer con los residuos especiales (el $81,1 \%$ ), ni procura que su proveedor les entregue las mercancías con el mínimo envoltorio posible (el 57,6\%). Como colofón, una gran parte de los comerciantes (un 86,8\%) dice conocer las causas del aumento de la temperatura del planeta, e incluso que su actividad puede estar contribuyendo al cambio climático (el 54,3\%), pero la gran mayoría (el 83,1\%) dice que no estaría dispuesta a firmar un documento comprometiéndose a realizar alguna acción, voluntariamente elegida por ellos, para minimizar la huella de carbono de su establecimiento (solo el 16,9\% dice que sí).

En definitiva, se observa una serie de contradicciones evidentes en las respuestas de los comerciantes a esta encuesta, lo cual hace difícil interpretarlas. Por ello, en el presente texto, nos planteamos la conveniencia de utilizar una metodología alternativa que permita desentrañar las contradicciones aparentes, así como averiguar información y propuestas para que la A21L pueda ayudar a los comerciantes a avanzar hacia la sostenibilidad. 


\section{El método STAVE}

Una gran parte de la investigación social sobre sostenibilidad se ha dedicado a identificar y medir las actitudes de la población respecto a temas relacionados con el medio ambiente o sobre su disposición a adoptar unos comportamientos más sostenibles. La encuesta mencionada en el apartado anterior lo ejemplifica bastante bien, pero este tipo de aproximación metodológica es problemática, puesto que implica presuponer que existe algún tipo inequívoco de conocimiento que se encuentra en la mente de las personas encuestadas y que el cuestionario permitirá captar. Sin embargo, a menudo, lo que se obtiene son las respuestas que aquellas personas consideran adecuado ofrecer a un investigador que les plantea las preguntas en un contexto muy connotado (en términos de sostenibilidad, etc.). De alguna manera, este tipo de estudios no suele cubrir la brecha existente entre los razonamientos prácticos de las personas encuestadas y las respuestas que estas dan ante preguntas descontextualizadas de sus experiencias cotidianas. En dicho entorno, es bastante probable que las respuestas que indiquen comportamientos poco sostenibles sean percibidas como socialmente inaceptables para muchas personas. Así, las respuestas a un cuestionario, más que como opiniones o actitudes, podrían ser interpretadas más bien como aspiraciones de dichas personas, con lo cual se mantendría una brecha o gap entre lo que manifiestan en sus discursos y sus prácticas relativas a la sostenibilidad.

Aquí presentamos una metodología llamada STAVE (Systematic Tool for Behavioural Assumption, Validation and Exploration), que, entre otras cosas, pretende servir para disminuir o cubrir el gap entre lo que la gente dice y lo que la gente hace en términos ambientales y de sostenibilidad (Horlick-Jones y Prades, 2014; Prades et al., 2015)3. A modo de ilustración, en el presente texto, se presentarán tan solo los resultados de una de las aplicaciones del método en España, relativa a las prácticas cotidianas de sostenibilidad de una pequeña muestra de comerciantes y su posible relación con la A21L.

La metodología está basada en un trabajo con los actores por dos vías:

- Por un lado, se procura establecer una implicación con las instituciones administrativas mediante un procedimiento de investigación-acción. Para ello, el método prevé el análisis de las políticas ambientales objeto de estudio, así como una serie de reuniones con los responsables institucionales, todo ello con el objetivo de entender con qué criterios se toman las decisiones, sus supuestos subyacentes y las asunciones implícitas sobre las personas

3. Este método se ha desarrollado en el marco del proyecto europeo PACHELBEL (Policy Addressing Climate Change and Learning about Consumer Behaviour and Everyday Life) (FP7, 2010-2012, grant agreement $n .^{\circ} 244024$ ) y ha sido probado en seis países europeos (Alemania, España, Francia, Reino Unido, Rumanía y Suecia). Los temas analizados variaron en cada país, en función de las necesidades de las administraciones públicas colaboradoras, y fueron el consumo doméstico de energía, la movilidad, el uso de redes eléctricas y los contadores inteligentes la toma de decisiones para el aislamiento térmico de edificios y para el recambio de electrodomésticos. 
objeto de aquellas políticas. Entender el proceso de formulación de políticas desde el interior (acceso al backstage) (Horlick-Jones y Rosenhead, 2002, 2007), así como el papel del conocimiento en contextos organizacionales (procesos de presentación, discusión y acuerdos sobre la realidad; razonamientos prácticos; uso de diferentes formas y fuentes de conocimiento; etc.) (Horlick-Jones, 2005; Horlick-Jones et al., 2007). Mediante este proceso, se pretende obtener un conocimiento bastante íntimo de los aspectos prácticos y cotidianos del trayecto de toma de decisiones en la formulación de políticas públicas.

- Por otro lado, se implementa un proceso de investigación con grupos deliberativos ciudadanos, con el objetivo de investigar sus desarrollos de aprendizaje y razonamientos prácticos, así como su percepción sobre el impacto de sus comportamientos de consumo y sobre el posible impacto de las políticas ambientales en sus vidas cotidianas. Se trata de un miniejercicio de participación ciudadana que incluye herramientas, como, por ejemplo: métodos de estructuración de problemas (Rosenhead y Mingers, 2001), espacios de aprendizaje colaborativo (Horlick-Jones, 2008) o diarios (Alaszewski, 2006; Zimmerman y Wieder, 1977). El proceso en su conjunto está diseñado para obtener información sobre los aspectos prácticos del comportamiento de las personas en su vida cotidiana y su relación con la sostenibilidad.

La deliberación de grupo está basada en una modalidad híbrida del grupo de discusión, combinada estratégicamente con una serie de herramientas (tool kit) diseñadas para promover una reflexión iterativa sobre diferentes tipos de discursos generados por las personas participantes. Los componentes del método incluyen el uso de mapas de ideas (oval maps), que permiten realizar la representación visual de las ideas del grupo, a la vez que proporcionan un registro acumulativo y flexible de las discusiones, un conjunto de materiales de estímulo que incluye noticias simuladas y viñetas, un ejercicio de asignación de recursos que permite priorizar las ideas y las propuestas surgidas en el grupo, así como una serie de cuestionarios breves para provocar la identificación personal con diferentes aspectos relacionados con la sostenibilidad. Se preparan, además, actividades fuera del equipo, como una serie de diarios personales diseñados para vincular las prácticas cotidianas con las conversaciones grupales.

A partir de uno o varios grupos de discusión (de entre 8 a 10 personas cada uno), se ha diseñado una secuenciación de dichas herramientas a lo largo de un período de un mes, incluyendo tres reuniones colectivas (una al principio, otra hacia la mitad y otra al final del proceso), combinadas con la realización de dos etapas de diarios personales por parte de las personas participantes (durante las semanas inmediatamente posteriores a la primera y a la segunda reuniones) (figura 1). Mediante esta aproximación metodológica, se pretende generar discusiones de las que puedan emerger discursos y visibilizar prácticas y acuerdos socialmente compartidos por las personas participantes. Los breves cuestionarios, administrados al principio y al final del proceso, tienen la función de provocar un contraste entre las opiniones individuales y las evidencias 
Figura 1. Secuencia de la aplicación del método STAVE

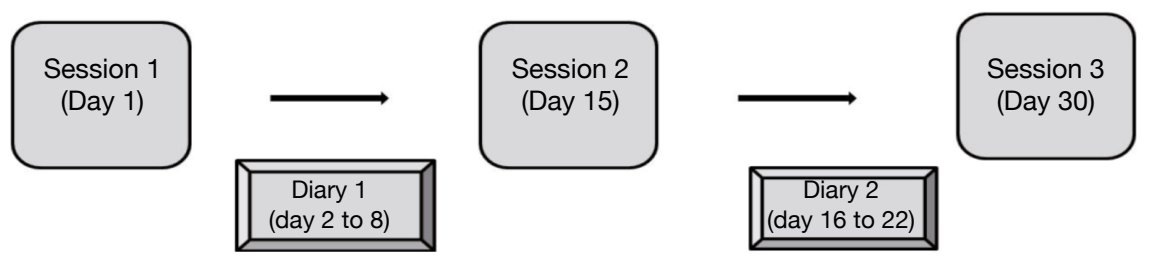

Fuente: Prades et al., 2015.

recogidas a lo largo del mismo, lo cual promueve una confrontación periódica y reflexiva de las personas con sus propias argumentaciones, en un proceso iterativo que permite dibujar puentes entre lo que dicen que piensan y lo que (dicen que) hacen en su vida cotidiana en cuanto a la sostenibilidad.

El método no solo está dirigido al análisis de los discursos y a las prácticas de una población, sino también a la interacción de esta población con alguna política pública por la sostenibilidad, lo cual implica hacer también un análisis de la institución o de la administración pública que ha diseñado dicha política (el policy making). Aquí vamos a aplicar el método STAVE en el marco de una investigación sobre la A21L de Barcelona.

\section{El estudio de caso: ¿Cómo puede contribuir la A21L de Barcelona a conseguir que el pequeño comercio local avance hacia la sostenibilidad ambiental?}

En el presente texto, mostramos los resultados del caso de la sostenibilidad en el sector del pequeño comercio, uno de los casos estudiados en el marco del proyecto PACHELBEL, y su relación con las políticas de la Agenda 21 Local de Barcelona. La investigación parte de una demanda de la oficina técnica de la A21L de Barcelona, que ha ido implementando un programa de implicación de entidades, instituciones y empresas para el cumplimiento de los objetivos de sostenibilidad (ver, más arriba, la información sobre el Compromiso ciudadano por la sostenibilidad). Como se ha dicho anteriormente, se parte de la idea de que los comerciantes podrían ser unos actores clave para mejorar la sostenibilidad en la ciudad, pero, por sus características, conforman un colectivo poco accesible para los programas de la A21L. A pesar de que se ha realizado alguna encuesta para conocer su posición en cuanto a la sostenibilidad, los resultados han sido superficiales y algo desconcertantes, por lo que se sigue desconociendo la realidad cotidiana del comercio local, lo que dificulta diseñar una política adecuada dirigida a este colectivo. Aunque el tema no ha sido demasiado tratado en la bibliografía, se encuentran estudios como el de Sancassiani (2005), quien realiza un análisis exhaustivo de 535 A21L italianas y observa que la participación de los comerciantes ha sido muy escasa en la gran mayoría de procesos participativos y de proyectos vinculados. 
En este contexto, el objetivo de la investigación consistió en averiguar qué percepciones y experiencias tienen los comerciantes respecto a la sostenibilidad de sus actividades comerciales, así como qué expectativas tienen sobre cómo la A21L les podría ser de utilidad.

De acuerdo con el protocolo del método STAVE, el estudio realizado ha supuesto un abordaje metodológico de doble vía:

\section{a) Análisis de los supuestos en los que descansa la A21L de Barcelona}

Por un lado, se ha estudiado la A21L de Barcelona, sus supuestos de partida y sus asunciones implícitas sobre los ciudadanos y las entidades que son objeto de sus políticas, programas y actividades. Para ello, se ha analizado la estructura, el organigrama y el funcionamiento de la A21L de Barcelona, se ha realizado un análisis documental de los principales textos generados en su seno y se ha llevado a cabo una serie de entrevistas en profundidad (30) a una muestra de entidades asociadas al programa Acción 21 (y firmantes del Compromiso ciudadano por la sostenibilidad). La muestra de 30 entrevistas se estructuró en función del tamaño de la entidad, de si tenía relación con temas ambientales o no y de si había elaborado o no un plan de acción de acuerdo con el compromiso firmado con la A21L. Además, se entrevistó también a 3 de los responsables institucionales de la A21L y se asistió a varias de sus reuniones de trabajo (a modo de observación participante).

\section{b) Análisis de las percepciones, los discursos y las prácticas de los comerciantes}

Por otro lado, se han analizado las percepciones, los discursos y las prácticas de una muestra de comerciantes en cuanto a sus relaciones con la sostenibilidad y con la política pública en cuestión (la A21L). Para ello, se ha aplicado el método STAVE a un grupo formado por 8 comerciantes (o representantes de pequeños comercios) de un barrio de Barcelona (Sagrada Familia). El reclutamiento se ha hecho a través de un gremio de comercio de dicho distrito de la ciudad. En la tabla 1, se muestran las características de las 8 personas participantes en el proceso.

Las reuniones de grupo se hicieron en el año 2011 en el local municipal del Centro Cívico Sagrada Familia y tuvieron una duración de dos horas (de

Tabla 1. Descripción de la muestra de comerciantes

\begin{tabular}{lllcc}
\hline Tipo de comercio & Relación laboral & Local & Sexo & Edad \\
\hline Heladería & Propietario (autónomo) & Propio & $\mathrm{H}$ & 60 \\
Telefonía móvil & Propietaria (franquicia) & Alquilado & $\mathrm{M}$ & 38 \\
Cosmética & Propietario (autónomo) & Alquilado & $\mathrm{H}$ & 46 \\
Cortinas & Propietario (SL) & Propio & $\mathrm{H}$ & 51 \\
Tienda de ropa & Propietaria (autónoma) & Propio & $\mathrm{M}$ & 36 \\
Joyería & Propietaria (SL) & Propio & $\mathrm{M}$ & 57 \\
Toallas y ropa de hogar & Propietaria (SL) & Alquilado & $\mathrm{M}$ & 31 \\
Asesoría administrativa & Propietaria (SL) & Alquilado & $\mathrm{M}$ & 53 \\
\hline
\end{tabular}

Fuente: elaboración propia. 
14 a 16, el único horario que iba bien a los comerciantes asistentes). La participación fue remunerada con 100 euros por persona, que incluían la asistencia a tres reuniones y la elaboración de diarios durante dos semanas. La primera tuvo lugar el 16 de junio; la segunda, el 29 de junio, y la tercera, el 13 de julio. Durante las reuniones, se aplicaron varias técnicas de dinamización diseñadas especialmente para la ocasión, con el objetivo de generar un debate reflexivo. Después de haber realizado la primera y la segunda, las personas participantes elaboraron un diario durante un plazo de una semana en cada ocasión y diariamente lo enviaban al equipo investigador. En el caso de varias personas, los diarios se hicieron por vía telefónica. En ellos, se planteaban tres cuestiones básicas: qué habían hecho aquel día que tuviera relación con la sostenibilidad, qué habían oído o visto relacionado con dicho tema y si habían hablado con alguien sobre él. Una selección de ideas y frases provenientes de los diarios fueron llevados a las discusiones de grupo para generar una mayor reflexividad, confrontar a las personas con sus propias opiniones y forzarlas a reafirmar o a cuestionar el sentido de lo que decían, lo cual hacía aflorar los diferentes niveles de discursos y sus argumentos y razonamientos para defenderlos.

\section{Resultados}

\subsection{Supuestos subyacentes a la A21L de Barcelona}

El análisis realizado muestra que la A21L descansaba sobre dos supuestos principales (compartidos por los responsables políticos del programa): la enorme dificultad para generar cambios estructurales a corto plazo a fin de avanzar hacia la sostenibilidad, lo que les llevaba a intentar provocar cambios a largo plazo mediante estrategias de educación ambiental (y difusión de «buenas prácticas»), y el supuesto de la voluntariedad para avanzar hacia la sostenibilidad (Espluga et al., 2012).

\section{a) El supuesto de la imposibilidad (o gran dificultad) de llevar a cabo cambios estructurales y la apuesta por la educación ambiental y la difusión de ejemplos de "buenas prácticas»}

Se observa que, para los responsables de diseñar la A21L, las políticas para la sostenibilidad ambiental tropiezan con una serie de obstáculos estructurales, contra los cuales, desde su punto de vista, es difícil incidir desde su departamento, como, por ejemplo: los modelos predominantes de consumo de masas, la fragmentación institucional que dificulta coherencia en las políticas públicas, etc. Por ello, desde la A21L se apuesta por focalizar sus (limitados) recursos y esfuerzos principalmente en acciones de información, comunicación y educación ambiental.

Uno de los ámbitos donde esto se produce es, por ejemplo, en la promoción de la educación ambiental en las escuelas (como estrategia de difusión básica). Otro es en la red de firmantes del Compromiso ciudadano por la sostenibilidad, donde la estrategia esencial consiste en hacer visibles las «buenas prácticas» que algunas de las entidades, instituciones y empresas de la ciudad ya están 
llevando a cabo, con la esperanza de que generarán mucha más credibilidad que los mensajes que pueda emitir el propio Ayuntamiento. En este sentido, se espera que la difusión de «buenas prácticas» multiplique los efectos de las acciones de información, comunicación o sensibilización.

En el fundamento de esta estrategia, se encuentra el supuesto de que los hábitos de consumo de la población están muy influidos por un modelo publicitario típico de una sociedad de consumo de masas, lo cual comporta serias dificultades para avanzar hacia la sostenibilidad. Sin embargo, al mismo tiempo, los responsables de la A21L sostienen que la publicidad institucional no tiene el mismo efecto, lo cual despierta dudas sobre las posibilidades de las organizaciones públicas para emitir mensajes convincentes sobre sostenibilidad. De ahí que hayan optado por diseñar un programa en el que los mensajes sobre cómo actuar para avanzar hacia la sostenibilidad sean vehiculados por entidades que ya realizan «buenas prácticas». De esta manera, el mencionado programa Acción 21 se basa, principalmente, en la promoción y el mantenimiento de una red de empresas, entidades e instituciones a las que se dinamiza mediante la oferta de cursos formativos, conferencias, entregas de premios, etc., cuyo principal objetivo es crear espacios de encuentro y visibilizar las «buenas prácticas» generadas por la propia red.

\section{b) El supuesto de la voluntariedad}

Las acciones realizadas en el marco de la A21L (y, en particular, el programa Acción 21 o Barcelona + Sostenible a partir de 2012) parten de la idea de que buena parte de la población permanece (voluntariamente o no) al margen de la información sobre sostenibilidad ambiental. Se considera que es difícil de hacerla llegar al público en general, porque vivimos en una sociedad saturada de información y no es fácil encontrar los canales adecuados para comunicarse con el público acerca de estos temas, sobre todo cuando se trata de un tipo de información que pone el acento en el ahorro, la reutilización, etc., con propuestas poco adaptables a la lógica de la sociedad de consumo.

De acuerdo con el análisis efectuado, desde la A21L, se clasifica a la población de la ciudad en función de su actitud potencial ante la información ambiental, clasificándola en tres tipologías:

- Hay personas a las que no les llega (no pueden acceder o, si lo hacen, no la pueden entender).

- Hay personas a las que sí les llega, pero prefieren no saber nada del tema.

- Hay personas a las que sí les llega y actúan en consecuencia (procurando la mayor sostenibilidad y el máximo respeto ambiental posibles).

Los programas de la A21L focalizan sus esfuerzos sobre todo en este último tipo de personas. Desde la A21L, se parte del supuesto de la futilidad de proveer información a gente poco predispuesta a adquirirla, por lo que se prefiere dedicar los recursos a quien ya está receptivo a aceptar los datos y los conocimientos que el Ayuntamiento puede facilitar. 
Esto se traduce en la enorme importancia de la «voluntariedad», pues solo se implica quien quiere. Por ello, el programa Acción 21, dedicado a la implicación de entidades, instituciones y empresas de la ciudad, apelaba continuamente a la voluntariedad, sin plantearse en ningún momento acciones obligatorias ni penalizadoras. La misma orientación se sigue en el nuevo programa Barcelona + Sostenible.

En este sentido, es interesante observar que no todas las entidades firmantes del Compromiso ciudadano por la sostenibilidad llevan a cabo las «buenas prácticas" prometidas, puesto que solo cerca de una tercera parte ha hecho público su plan de acción (en Espluga et al., 2011, se analizan con más detalle los motivos), pero el diseño de esta política pública no permite obligar a nadie a adoptar unas actitudes más sostenibles.

\subsection{Percepciones, discursos y prácticas de los comerciantes sobre la sostenibilidad y la $A 21 L$}

En este apartado, se describen y se interpretan los resultados obtenidos mediante la aplicación del método STAVE a la muestra de comerciantes. Aunque el método genera una gran cantidad de información (proveniente de las discusiones de grupo, de los ejercicios realizados en las sesiones colectivas, de los diarios individuales, de los cuestionarios, etc.), aquí se presentan resultados de manera agrupada y sintética, sin especificar la técnica de la que proviene cada idea o reflexión. Aunque se trata de un solo equipo compuesto por ocho personas, el proceso de reflexión realizado es suficientemente intenso como para hacer aflorar una gran cantidad de información ampliamente argumentada por los propios protagonistas. Además, se trata de un estudio exploratorio cuya función en el presente texto pretende mostrar el funcionamiento del método STAVE y los resultados que se pueden obtener del mismo.

En los resultados, en primer lugar, se tratan sus percepciones sobre la sostenibilidad en general, un concepto que tienden a relacionar con temas económicos (principalmente, en forma de costes). Más adelante, se explican los principales ámbitos en los que perciben que podrían incidir desde su actividad cotidiana (sobre todo, temas de energía y residuos). Posteriormente, se abordan las percepciones que tienen respecto a su capacidad para actuar de manera más sostenible y los obstáculos que encuentran para ello. Finalmente, se comenta cómo la A21L en particular y el Ayuntamiento en general podrían serles de utilidad para avanzar hacia la sostenibilidad.

\subsubsection{Percepciones sobre la sostenibilidad}

De entrada, los comerciantes participantes en el grupo de estudio procuran mostrar una predisposición positiva hacia la sostenibilidad. Hay que advertir que estas personas han sido reclutadas a través de una asociación de comerciantes que es firmante del Compromiso ciudadano por la sostenibilidad promovido desde la A21L, lo cual podría hacer suponer un cierto sesgo favorable a las preocupaciones ambientales. Sin embargo, se observa que la mayoría de 
los participantes manifiesta desconocer el programa A21L y que ha decidido participar en nuestra investigación porque cree que el tema es de su interés. En algunos casos, lo justifica apelando a motivos familiares (uno de los participantes afirma que un hijo suyo ha cursado estudios de ingeniería ambiental). De hecho, a juzgar por las expresiones de los participantes, parte de sus actitudes hacia la sostenibilidad parecen estar influidas por el contexto doméstico. Así, por ejemplo, hacen referencia a cómo los hijos pequeños aprenden en la escuela comportamientos y hábitos sostenibles que luego transmiten a los miembros del hogar. Algunos de los participantes manifiestan conocer ciertas «buenas prácticas» sostenibles gracias a la presión infantil (e incluso se sorprenden de que los pequeños hayan adquirido el hábito de ahorrar agua o energía, o de separar residuos, etc., puesto que perciben dichas actitudes como algo ajeno o incluso un poco excéntrico) (fragmento 1).

Fragmento 1. Segunda reunión de grupo

$\mathrm{H}$ : [¿Que si] los hijos influyen en esto? Vamos, totalmente.

M: Sí, sí.

$\mathrm{H}$ : Porque en los colegios sí que los insisten continuamente con todo este tipo de cosas. Y eso se transmite.

M: Sí, sí.

$\mathrm{H}$ : Se transmite. Yo me he quedado alucinado. Yo tengo una nieta de dos años y le das un papel y lo va a tirar a la papelera. $\mathrm{O}$ sea, que no te lo tira, es verdad, es verdad. Oye, lo tira a la papelera o va a la basura con él. [...] No, no, es que me he quedado alucinado. $\mathrm{O}$ sea, las veces que le habrán insistido, eh. Imagínate una niña tan pequeña, lo que le habrán dicho para que vaya a la papelera, digo yo.

El hecho de que encuentren sorprendente que los niños lleven a cabo prácticas sostenibles puede ser un indicio de que existe una cierta distancia entre la conciencia ambiental de los participantes y sus comportamientos efectivos.

Entre las personas participantes se da una interesante discusión sobre qué es un producto ecológico o natural. La mayoría de ellas son escépticas y llegan a sostener que se trata sobre todo de un discurso publicitario para vender más caros ciertos productos (incluso sospechan que debe haber mucha picaresca) (fragmento 2). De alguna forma, la sostenibilidad se tiende a considerar también como una especie de «moda» que atrae cada vez a más gente ("es lo que más se lleva ahora»).

Fragmento 2. Tercera reunión de grupo

H: [...] o el poder conseguir ser más ecológico o más, más ecológico, más efectivo en el tema de las energías, pero como es más caro, generalmente es más caro, pues una parte o una gran cantidad de proveedores o de fabricantes utilizan la picaresca y te ponen allí cualquier etiqueta medio rara y los que lo hacen bien y correctamente es más caro, por lo cual hay un público que está dispuesto a pagar esto, pero la mayoría del público no está dispuesto a pagar el precio, por lo cual siempre vamos al problema económico. Y si no hubiera un problema económico, seguramente no habría un problema ecológico. Todo el mundo está concienciado, creo, nadie está por la labor. 
H: ¿A qué conclusión llegas? ¿A qué conclusión se llega con esto?

$\mathrm{H}$ : A la económica.

H: La mayoría de las cosas, lo que hacen es publicitar para poder vender más caro. ¿Y por qué es más caro lo mío? Ah, es que lo mío es...

\subsubsection{Aspectos clave para avanzar hacia la sostenibilidad}

Entre los condicionantes percibidos y argumentados por los comerciantes para avanzar hacia la sostenibilidad, aparecen los siguientes factores:

\section{a) Costes económicos}

Por un lado, se pone en cuestión la idea misma de «buenas prácticas» sostenibles. Se interpretan simplemente como formas razonables de no gastar recursos, de no generar residuos, etc. (de ahorrar dinero, en definitiva). De hecho, en los cuestionarios realizados en la primera reunión de grupo, se pedía que escribieran las tres palabras que intuitivamente más relacionaban con «consumo sostenible», y la que más se repitió fue ahorro. Por ello, tienden a equiparar las «buenas prácticas» al hecho de «tener menos gastos», algo que, según ellos, es lo que los comerciantes "deberían hacer siempre».

Sin embargo, al mismo tiempo, expresan otro discurso que se basa en que, a menudo, "lo ecológico sale más caro que lo que contamina», lo cual les sitúa en una cierta contradicción argumental. Su forma de resolverla es decir que la situación solo es anómala "de momento" y que en un futuro indeterminado será más caro lo no sostenible (fragmento 3).

Fragmento 3. Primera reunión de grupo

H: Está claro, pero si yo vendo un producto de 60 euros o 100 euros, pues claro, voy a poner una bolsita hasta de tela y..., pero claro, cuando son productos pequeñitos o productos..., te lo has de mirar, te lo has de pensar porque es un presupuesto...

M: Es como inversamente proporcional, o sea, lo que es ecológico, verde, lo que es de esto, sale más caro que lo que contamina, por decir algo.

$\mathrm{H}$ : Exacto.

$\mathrm{H}$ : De momento, de momento, eso es lo que pretenderíamos que eso fuese precisamente más barato, o sea, siempre, si tú vas a comprar una verdura, una fruta ecológica que dice "ecológica», que habría que verlo pero posiblemente sea verdad, tiene peor presencia y es más cara. Luego, hasta que esto no encaje bien..., es muy difícil hablar de lo ecológico y de lo otro... Y más cuando estamos en un momento de ajuste de precios.

De alguna manera, para ellos, la idea de sostenibilidad remite a algo que ven «normal» en un futuro indeterminado. Pero ellos viven en el presente, y aunque les gustaría orientarse hacia ese futuro, a lo largo de la discusión, también abundan en sus dificultades económicas para hacer la transición.

\section{b) Energía}

Al pensar en cómo avanzar hacia prácticas más sostenibles, las personas participantes dedican mucho espacio a la discusión sobre los costes de la energía y 
sus dificultades para disminuirlos. Desde su punto de vista, la iluminación de una tienda es muy importante, es un factor vital para el pequeño comercio. Aunque ponerla de bajo consumo sería lo ideal, perciben que ello requiere de una inversión muy elevada (que consideran «inasumible» en muchos casos), por lo que sugieren la necesidad de crear subvenciones, normativas de edificación más favorables, etc. Además, según ellos, en la actualidad, los comerciantes que deciden hacer modificaciones para adaptar sus instalaciones son penalizados con los impuestos de obras, pues estas son más caras.

En general, creen que gastan más energía en verano que en invierno, sobre todo a causa del aire acondicionado, y lo argumentan con experiencias cotidianas (fragmento 4). También explican que, aunque quieran poner el aire acondicionado a una temperatura más bien alta (para gastar menos), si entra mucha gente en la tienda, se ven obligados a bajarla, porque, si no, no se nota (con lo que inevitablemente aumenta el consumo eléctrico).

Fragmento 4. Primera reunión de grupo

$\mathrm{H}$ : Posiblemente sea mayor en verano que en invierno, porque yo creo que el dar calor es más barato que el dar frío.

M: No, pero el frío igual son cuatro días que tienes que encender. Por mí, ¡eh! Cuatro días.

M: Porque con las luces...

$\mathrm{H}$ : Es que, en invierno, con muy poco calienta y si procuras que no esté abierto, se mantiene. En cambio, en verano, el aire tiene que estar constante, a la que desenchufas o baja un poco, bum.

M: A parte de que dicen que si lo bajas y subes mucho se te estropea.

En definitiva, las personas participantes observan que sí tienen ciertas posibilidades de ahorrar energía ( $y$, por tanto, dinero), por ejemplo, regulando el aire acondicionado. Pero se advierte que no todo el mundo es consciente de ello, ya que hay que tener voluntad para monitorizarlo y calcularlo. De todas formas, aunque todos redujeran el consumo de energía, están convencidos de que ello no repercutiría en una disminución de su coste, pues tienden a creer que les subirían el precio de la factura en la misma proporción que hubieran reducido el consumo. Basan esta argumentación en su percepción de la gran opacidad del funcionamiento del sistema eléctrico y las empresas proveedoras.

Fragmento 5. Tercera reunión de grupo

¿Pero todos creéis que no se puede hacer nada para modificar el consumo?

M: Hombre, se puede hacer.

M: Algo se debe poder hacer.

$\mathrm{H}$ : Pero económicamente tampoco.

M: Pero que la inversión es cara es cierto.

H: Mira, yo estoy seguro, además. Quizás si todo el mundo, y vamos a ponernos en el supuesto de que todo el mundo hiciese que se consumiese menos energía, podríamos consumir menos energía. Pero no pagaríamos menos, por lo cual alguien se beneficiaría y no seríamos los que ahorrásemos la energía. Eso es lo que yo pienso y ahí es donde está el problema, porque 
cuando yo ahorro agua y trato de consumir menos agua, el recibo me viene igual, cuando ahorro gas yo pago lo mismo, aunque trate de ahorrar... Es que no veo casi ninguna diferencia.

$\mathrm{H}$ : Ahora te lo subirán un $10 \%$. Ya no te vendrá igual.

$\mathrm{H}$ : Es que el esfuerzo que tú haces de ahorro no encuentra una contraprestación a que te salga más barato, y eso es lo malo, que debería salir más barato si tú pones algo, pues no.

M: Es que los impuestos...

H: Claro, la compañía eléctrica dice: «Ah, facturo menos. Habrá que subir el precio. Porque facturo menos, claro».

\section{c) Residuos}

Se observa que la sostenibilidad la relacionan sobre todo con el tema del «reciclaje». De algún modo, la generación de residuos la equiparan a la realización de actividad (económica, productiva, etc.), hasta el punto de compartir la idea de que «quien no genera residuos, no existe».

Las personas participantes observan que, durante la actividad comercial, se produce una cantidad creciente de residuos, especialmente por el elevado uso de envoltorios y envases. Por ello, tienden a considerar que sería más sostenible hacer retornables muchos envases o productos. Por ejemplo: sugieren que se podría compensar al cliente por electrodomésticos retornados al comprar uno nuevo. También comentan que, tarde o temprano, se tendrá que volver al envase retornable, aunque hay discrepancias sobre si en las condiciones actuales sería rentable o no.

Comentan que muchos productos llevan más envases de los necesarios porque así lo obliga la legislación (ponen el ejemplo de las bebidas o de los alimentos que tienen que estar envasados individualmente y bien etiquetados por cuestiones de seguridad sanitaria). No obstante, se comenta que otro tipo de productos (como, por ejemplo, cartuchos de tinta) llevan demasiados envoltorios que se podrían reducir. No obstante, hay quien sospecha que incluso estos productos, si los han envasado así, será porque o bien no hay más remedio (legislación) o porque es como sale más rentable a los fabricantes. Se niegan a aceptar que, pudiendo poner menos envases, haya empresas que quieran poner más de los imprescindibles, que quieran pagar más de lo necesario. En este sentido, se hace referencia a la importancia de la publicidad, de la imagen de marca, que se transmite a través de los envoltorios, y se alude al argumento de que una estrategia publicitaria puede ser rentable aunque no sea sostenible desde el punto de vista ambiental.

Otro aspecto relacionado con los residuos es su adecuada selección y separación. De entrada, todos los participantes dicen conocer cuáles son las prácticas correctas de gestión de residuos en su comercio, y aseguran hacer muchos esfuerzos por separar y recoger de manera adecuada los que generan. Sin embargo, a lo largo del proceso de investigación, aparecen contradicciones evidentes entre sus argumentos y las experiencias relatadas.

Por ejemplo: tras afirmar la necesidad de seleccionar y reciclar bien los residuos, uno de los comerciantes dice tener problemas para hacerlo correctamen- 
te, por lo que finalmente reconoce que prefiere ignorar las normas de selección y depositarlos todos en el mismo tipo de contenedor (genérico) (fragmento 6).

Fragmento 6. Segunda reunión de grupo

H: A ver, por último, un par de cosas sobre el reciclaje de esto, de... envases y tal... Todos los días pienso en cómo reciclar los envases de vidrio. En casa, lo reciclo bien, cada cosa en su contenedor, pero en el comercio no hay manera. Por un lado, tengo poco espacio [en el local] para acumular botellitas y, por otro lado, si voy a tirarlos al contenedor de vidrio, me paso media mañana, porque hay que tirarlos uno a uno. Yo genero muchos y el contenedor está bastante lejos... No me sale a cuenta.

H: Y, si tiene líquido, encima corres el riesgo de mojarte [...] todo allí... botellín a botellín. 150 botellines. ¿Cómo metes 150 botellines en un momento allí?

H: En mi casa, somos 6 y ya estás un rato, pero tú, con 150, agárrate.

H: Bueno, eso como mínimo, estás allí metiendo...

$\mathrm{H}$ : Y si van con..., a veces llevan un poco así de líquido, tiene..., digo yo, tiene que haber algo...

Es preciso destacar que este participante, como otros, en la primera reunión de grupo mantuvo su imagen de persona ambientalmente responsable, pero las preguntas que el equipo investigador le hizo a través del diario telefónico permitieron hacer aflorar dicho comportamiento poco sostenible, que luego, cuando fue trasladado a la reunión de grupo, permitió debatirlo colectivamente y entender sus razonamientos prácticos. El proceso iterativo propiciado por el método STAVE, que confronta a las personas con sus propias opiniones varias veces a lo largo del proceso, ha permitido hacer aflorar ciertos comportamientos transgresores de las normas de selección de residuos, observar la distancia entre sus expresiones y sus prácticas y visibilizar la lógica subyacente a dichos comportamientos.

Otro aspecto clave de las relaciones que entablan con los residuos es su proceso de recogida. En este sentido, se reclaman mejoras en los contenedores de papel y cartón, aunque las personas que más insisten en ello son las que menos parecen utilizarlos. Algunos comerciantes dicen contar con un servicio especial (municipal) que les recoge periódicamente los cartones que generan. Otros dicen no saber que dicho servicio existe y prefieren tirarlos en el contenedor genérico, y como excusa se expresa la incomodidad de guardar y trasladar las cajas grandes de cartón a los contenedores azules (fragmento 7).

Fragmento 7. Segunda reunión de grupo

M: Y sabes que a nosotros no nos recogieron. Le mandé un mail y, al otro día: «Lo siento, mañana pasaremos».

$\mathrm{H}$ : Pero eso mismo que, por ejemplo, si te lo recogen al lado va muy bien, pero si lo tienes que meter tú al contenedor... Tú un paquete así de cajas que has prensado previamente ahora las sueltas del cordón donde las llevas arrastradas. Sácate el cordón, una a una, y empieza a meterlas también allí al cacharrito que tienen. Yo te lo digo porque es un incordio echar las 
cosas, pues a mí me sale mucho más rápido al «basurón» grande. Para allá, venga, claro.

$\mathrm{H}:$ ¿Y a vosotras como es que os lo pasan a recoger?

M: A nosotras nos lo pasan... A mí me lo pasan a recoger cada martes, jueves y sábado. A las ocho y media de la noche.

$\mathrm{H}$ : Pasa que, a mí, eso no me funciona. Tengo poco. Puedo tener, como mucho, una caja y no muy grande al día. Entonces, tampoco...

$\mathrm{H}$ : El cartón es lo que os pasan a recoger.

$\mathrm{H}$ : Y si no vienen, les mandas un mail y te lo contestan, y vienen al otro día y te piden disculpas.

Se observa que, debido a la falta de espacio en sus locales, algunos comercios dejan los envases en la calle con varios días de antelación, pero, a menudo, estos cartones acaban desparramados por la acera a causa de actuaciones incívicas de algunos ciudadanos. Por ello, quien no puede guardarlos hasta el día adecuado, prefiere tirarlos a los contenedores de papel o a los genéricos. También se comenta que estos contenedores son víctimas de numerosos robos, pues ven que cada vez hay más gente que se lleva los papeles y cartones que contienen. Se observa una cierta ambivalencia a la hora de valorar estos comportamientos, pues, por un lado, se considera que contribuyen a realizar el reciclaje, pero, por otro, les molesta que lo hagan, porque no se lo llevan todo y a menudo ensucian la calle. Incluso uno de los comerciantes asegura haber sido multado porque cajas con la etiqueta de su comercio fueron encontradas tiradas en la calle después de haberlas depositado correctamente. Para evitarlo, hay quien sugiere crear la figura de los "vigilantes cívicos», una especie de voluntarios o similar para controlar a la gente que se lleva los cartones de los contenedores. El «vigilante cívico» también estaría al tanto (supuestamente) de otras cosas (de quien no recoja los excrementos de perro, de que no haya niños por la calle en horario escolar, etc.). No obstante, a lo largo de la discusión, los participantes se muestran algo escépticos con la idea e incluso hay quien pregunta: «¿Quién vigilará a los vigilantes?».

De hecho, cuando, en la tercera y última reunión del grupo, las personas participantes hicieron el ejercicio de asignar recursos, consistente en atribuir puntos (o recursos monetarios hipotéticos) a diferentes propuestas para mejorar la sostenibilidad, la del vigilante cívico quedó sin puntuar. Es decir, resultó ser un elemento lógico (que quedaba bien decir) en el contexto de la discusión colectiva, pero, en realidad, ninguno de los participantes era partidario de ello. De alguna manera, la petición de dicha figura parece reflejar la sensación de indefensión de los comerciantes ante actuaciones poco cívicas de otra gente (o de otros comerciantes), pues se sienten en un entorno y en un ámbito (el de la sostenibilidad) en el que perciben que suelen pagar justos por pecadores.

Para avanzar hacia la sostenibilidad, consideran que es mejor premiar que castigar. Perciben que, para castigar, hay que crear un cuerpo de vigilantes y toda una infraestructura para vehicular las penalizaciones, además, creen que eso sería demasiado caro. Por ello, sostienen que es preferible dar incentivos a 
los comerciantes para que puedan mejorar. Esto encaja relativamente bien con los supuestos de voluntariedad subyacentes a la A21L de la ciudad.

\subsubsection{Capacidades para avanzar hacia la sostenibilidad}

En las respuestas al cuestionario (pasado en la primera reunión de grupo), la mayoría de comerciantes manifestó tener una elevada capacidad de influir en su entorno para provocar cambios favorables hacia la sostenibilidad (contestaron con una media de 5,8 puntos sobre una escala de 6 a la pregunta: «iPuedo llevar a cabo acciones para que el consumo sostenible aumente?»). Sin embargo, al confrontarlos con las opiniones y con las prácticas reseñadas en sus diarios y luego debatirlos en las sesiones de grupo, reconocieron que su capacidad era más bien limitada, por varios factores:

\section{a) Factores relacionados con la dependencia de fabricantes y proveedores}

Los residuos se generan sobre todo por los envases y los embalajes de los productos que venden, pero los comerciantes dicen que no pueden cambiar gran cosa, porque ello depende de las empresas que se los mandan. En este sentido, su poder de decisión es mínimo, puesto que quienes toman estas decisiones son más bien sus proveedores y fabricantes. A lo largo de las sesiones de grupo comentan varias experiencias sobre cómo les proporcionan demasiados embalajes (fragmento 8).

Fragmento 8. Primera reunión de grupo

M: [...] El fomento del reciclaje estará siempre en función de la facilidad que se le dé para efectuar ese reciclaje correctamente, pero esto tiene que ponerse en la parte donde toca, no en los botiguers, que pueden hacer una parte pequeña. El fomento del reciclaje siempre dependerá de los envases que se pongan [en] los productos, ¿no? No aquí, el comerciante ya lo tiene tal cual viene y lo tiene que comprar así. Tener que elegir entre una cosa que está en un embalaje o en otro es bastante difícil. Tú vas a la más barata, a la que más te interesa, a la que tiene mejor vista, etc. El embalaje es una parte muy mínima en la que tú te fijas a la hora de comprar, luego esto debe de estar promovido a otros niveles. Los comerciantes aquí poco tienen que hacer. Ya quisiéramos reducir los costes del transporte. A mí me vienen y me dan lo que me dan.

Por ello, desde su punto de vista, sería más conveniente influir o presionar (o dar incentivos) a estos proveedores y fabricantes para que actuaran de manera más sostenible. En este sentido, se dice que ya existen certificaciones ambientales, etc. que pueden ayudar a avanzar en esa dirección, aunque también desconfían de estas estrategias, puesto que perciben que, a menudo, solo se traducen en un cumplimiento formal.

Se habla también de algunas experiencias de comerciantes que se agrupan entre ellos para exigir ciertas condiciones a sus proveedores (a través de una central de compras), y sugieren que podría ser una buena fórmula para presionar a los fabricantes a avanzar hacia la sostenibilidad. Sin embargo, parece que no todos pueden hacerlo, pues hay comerciantes que ven muy difícil unirse con 
otros de su mismo sector, pues son su competencia directa y suelen mantener relaciones de desconfianza mutua. No obstante, se reconoce que sería una iniciativa interesante, por ejemplo, para comprar bombillas de bajo consumo, bolsas reciclables, etc. Incluso hay quien propone que el Ayuntamiento haga este papel a fin de ayudar a que los pequeños comercios puedan proveerse de este modo, lo cual propiciaría unas mayores garantías de poder llevar a cabo «buenas prácticas» ambientales.

\section{b) Factores relacionados con el modelo de franquicia}

Algunos de los comercios representados en el grupo funcionan mediante un sistema de franquicia. La discusión permite observar que determinados modelos de franquicia pueden constituir también un obstáculo para avanzar hacia la sostenibilidad, ya que imponen sobre los comerciantes determinadas pautas a veces poco sostenibles (como tener más iluminación de la necesaria, etc.) (fragmento 9). Estas circunstancias revelan importantes restricciones a la autonomía del comerciante para tomar decisiones.

Fragmento 9. Primera reunión de grupo

M: Sí, lo de "obstáculos por la normativa de franquicia» lo he puesto yo, porque yo, por ejemplo, tengo que tener..., porque ellos me lo exigen..., pues tengo que poner, por decir..., veintiún ojos de buey, tantos de no sé cual... y, además, he de comprar de una marca tal de cual... Y entonces, cuando yo veo que es un día luminoso, yo quito algunos ojos de buey, porque es que, si no..., pues pago un montón de luz.

$\mathrm{H}:$ ¿Y eso tú no puedes hacer...? ¿Eso ya te viene impuesto?

M: Sí, ya viene impuesto, pero yo... Eso realmente cuando me dicen algo los pongo todos, y luego ya voy quitando cuando puedo, porque es que, si no, gasto mucha electricidad.

Aun así, deducen que el modelo actual debe ser el más eficiente en términos económicos, puesto que los proveedores, fabricantes y franquiciadores ya habrán hecho los cálculos para ofrecer sus productos de la manera que sean más rentables (la máxima productividad al mínimo coste). En cualquier caso, en estas situaciones, evidencian la limitada capacidad de los comerciantes para tomar decisiones y llevar a cabo prácticas más sostenibles.

\section{c) Factores relacionados con la presión del cliente}

Otro de los obstáculos con que se encuentran es que hay clientes que, sistemáticamente, les exigen envoltorios o bolsas adicionales, aunque el comerciante inicialmente no se la dé o se resista a dársela. Perciben que, mientras los grandes comercios pueden permitirse el lujo de no dar bolsas o incluso de cobrarlas a los clientes, los pequeños comercios creen que no pueden hacerlo por temor al riesgo a contrariar al cliente y perderlo («El cliente siempre tiene la razón»). Reconocen que deberían adoptar una posición común en este tema, pero no se ven capaces. Además, se sienten más vulnerables, porque, en el pequeño comercio, el cliente trata directamente con el dueño de la empresa. 
A pesar de que las personas participantes procuran poner en práctica estrategias para no tener que usar tantas bolsas de plástico en sus ventas y de que están convencidos de que hay que reducirlas, en su quehacer cotidiano, se encuentran con clientes que las piden (fragmento 10).

Fragmento 10. Segunda reunión de grupo

M: Los clientes piden bolsa.

M: Piden bolsa, piden bolsa.

M: Hasta para una reparación. A mí me traen un despertador que se lo sacan del cesto con migas, con todo lo que va en el cesto. Yo le hago lo que le tenga que hacer a este despertador. Se lo dejo allí y le digo, por ejemplo: «Son 3 euros», o lo que sea, y me dice: «¿Me dará una bolsa?». Para llevárselo. Te lo han traído en el cesto y te piden una bolsa para llevárselo, y este ejemplo vale para casi todo, jeh!

Sin embargo, también perciben que, a la larga, el dar bolsas será algo excepcional. Es algo que ya está promoviendo la gran superficie comercial y que sospechan que tendrá impacto a largo plazo en todo tipo de comercios.

La recursividad del método STAVE ha permitido hacer aflorar sus propias contradicciones, pues, aunque se quejan de estas actitudes del público, tras un cierto proceso de disimulo, acaban por reconocer que, cuando ellos adoptan el rol de clientes, se suelen comportar igual que los suyos. Así, por ejemplo, tras quejarse de que los clientes exigen demasiados envoltorios y bolsas, una de las participantes dice que, cuando ella va a comprar a un gran supermercado, lleva siempre su propia bolsa, pero, en cambio, cuando va a un comercio pequeño, pide que le den dos bolsas (que, para justificarlo en términos de sostenibilidad, dice utilizar para luego tirar la basura) (fragmento 11).

Fragmento 11. Segunda reunión de grupo

M: Yo te cuento una cosa que me pasa. En mi casa, lo reciclamos todo, porque, en el barrio, solo se puede reciclar, porque tiramos la basura por un agujero que es un contenedor neumático. Pero resulta que, en casa, no tenemos bolsas, porque si compras en el supermercado, en el supermercado ya no te dan, y para reciclar necesitas meter el plástico en una bolsa y llevarlo... Entonces, o compras bolsas o vas a los comercios pequeños y se las pides en tu compra, les dices: «Dame una bolsa, por favor», porque, si no, no tenemos bolsas. O sea, porque no tenemos donde meter los plásticos.

$\mathrm{M}: \mathrm{O}$ en un cubo. Yo, por ejemplo, en casa, tenemos un cubo que lo llenamos de plástico.

M: Sí, los papeles los llevamos en estas bolsas grandes reutilizables, pero los $[\ldots]$ plásticos, no. Los tiramos.

M: No, pero que yo estoy superacostumbrada a..., en supermercados, a llevar mi propia bolsa, pero, en un comercio pequeño, pido dos.

Desde su punto de vista, las alternativas son caras y creen que los comerciantes solo podrán ser más sostenibles actuando de manera coordinada (que todos se negaran a dar bolsas o que todos las cobraran). 
En cada una de las tres reuniones del grupo, se vuelve a expresar la preocupación por la presión del cliente, ya que, aunque el comerciante quiera reducir envases, el cliente siempre les puede exigir una bolsa o un embalaje más. Aun así, se dice que los comerciantes pueden también incidir en el tipo de bolsa que den al cliente. Aunque, a raíz de la discusión, ello parece solo ser cierto en los casos en que el producto vendido alcanza un cierto precio (se sugiere que, en productos muy baratos, no sería rentable dar una bolsa de papel o biodegradable, por ejemplo). Aun con todo, se dice que hay un sector de clientes que están concienciados y que podrían entender mejor estos cambios (hacia la sostenibilidad), por lo que quizá tendría sentido desarrollar alguna estrategia de acercamiento a este tipo de clientes (fragmento 12).

Fragmento 12. Primera reunión de grupo

M: Esto eran en el sentido de ventajas o cosas a aprovechar, soluciones conjuntas... Ya que estamos aquí varias asociaciones de comerciantes, pues aprovechar encontrar soluciones conjuntas o..., entre nosotros o con más gente, pues yo qué sé, para comprar bolsas, para... En realidad, lo de la gente de las tendencias lo pondría como una cosa positiva, porque yo, entre mis clientes, sí veo gente que está, no todos, pero gente que sí está concienciada con ello, y lo pide, ¡eh! Entonces, tiene su parte buena y su parte mala.

M: Yo sí que detecto gente que está preocupada por estas cosas...

H: Por lo tanto, sí que hay un sector de clientes que podrían entender mejor los cambios y, además, nos podríamos unir unos cuantos para hacer cosas. Por ejemplo: comprar bolsas de manera colectiva o bolsas de papel, espacio para contenedores...

\subsubsection{El rol de la A21L y de las politicas municipales}

Para finalizar, en este apartado, se apuntan algunas reflexiones de los comerciantes sobre posibles aportaciones que la A21L podría facilitarles, cosa que constituía uno de los objetivos clave de esta prueba piloto de aplicación del método STAVE. Hay que señalar que la metodología STAVE requiere conectar la esfera de la realidad cotidiana de los ciudadanos con la esfera de la toma de decisiones públicas, por lo que se prevén varios mecanismos de traslación de información de una esfera a otra. En este sentido, entre la segunda y la tercera reuniones del grupo de comerciantes, se realizó una devolución de la información generada hacia los responsables de la A21L, con el objetivo de observar cómo reaccionaban ante la información generada hasta aquel momento $\mathrm{y}$ recoger nuevas cuestiones a introducir en la última reunión colectiva, la cual se realizó en las instalaciones del Ayuntamiento con varias de las personas encargadas de la A21L.

Las reflexiones de los comerciantes sobre posibles acciones del Ayuntamiento se centran en la conveniencia de poder contar con ayudas económicas (subvenciones) para poder hacer la transición hacia un consumo más eficiente y sostenible, por ejemplo: para cambiar las instalaciones eléctricas por otras de bajo consumo, etc. Además, algunos comerciantes que están en locales de 
alquiler consideran que el gasto de estas reformas debería ir a cargo del propietario del local, pero perciben que es una lucha difícil de ganar, porque la legislación no les favorece.

Por otro lado, se pide que, en las normativas municipales (o generales), se exija que los nuevos edificios ya se construyan de manera más sostenible. Hay una cierta discusión sobre hasta qué punto dichas exigencias ya existen o no. Así como sobre hasta qué punto son convenientes, puesto que pueden aumentar el coste de los locales y requieren de un mantenimiento posterior presumiblemente más caro.

Otra propuesta que formulan es que, en lugar de dar subvenciones, el Ayuntamiento podría ofrecer «exenciones» a la hora de pagar ciertos tributos. Esta cuestión era de especial interés para los responsables de la A21L, pues, desde el consistorio, se promueven varias acciones políticas basadas en incentivos fiscales y subvenciones. Los comerciantes sugerían que, a pesar de que las subvenciones son más atractivas, en la práctica, saben que son más difíciles de obtener y, una vez concedidas, se suelen cobrar a muy largo plazo, por lo que observan que las exenciones tributarias pueden tener un impacto más efectivo en su cotidianidad.

En medio de la discusión, los comerciantes descubren que el Ayuntamiento ya está dando dinero a la asociación de comerciantes para hacer una campaña de información. Consideran que está bien vehicular estas iniciativas a través de las asociaciones de comerciantes, para animar a los negocios del barrio a asociarse y a difundir sus «buenas prácticas», cosa que coincide con la apuesta de la A21L.

Los participantes sostienen que el comercio local debería esforzarse por conseguir que los habitantes del barrio hicieran sus compras en el mismo. En este sentido, sugieren que el visibilizar sus buenas prácticas ambientales puede ser una forma de hacer más atractivo el comercio del barrio, algo para lo que la A21L podría serles de utilidad.

Un tema muy recurrente en este campo es el de los hipotéticos beneficios del reciclaje, que consideran que deberían visibilizarse más en el barrio. De ahí que reclamen algún tipo de compensación (para el barrio) por reciclar (por ejemplo: que se compre un columpio para el parque infantil, que se haga un club de jubilados, que lo pongan en la revista del Ayuntamiento, etc.). Por ello, en las discusiones de grupo, continuamente exigen mayor transparencia en todo el servicio de recogida de residuos urbanos, que es visto como demasiado opaco y genera una cierta desconfianza, lo cual desincentiva el llevar a cabo mejoras ambientales.

Los comerciantes hablan de los intentos de la A21L por ofrecerles información ambiental y sobre sostenibilidad, y consideran que la mejor forma de transmitírsela es a través de las asociaciones o gremios de comerciantes. Desde la A21L, se les intenta ofrecer también acciones formativas, pero se observa que los comerciantes son reticentes a las campañas de formación y educación, especialmente si van dirigidas a ellos (fragmento 13). En cambio, sí se muestran partidarios de que la A21L suministre información y haga acciones de sensibilización dirigidas a los clientes (y a la ciudadanía en general). 
Fragmento 13. Tercera reunión de grupo

H: No, no. Esa no es la cuestión. A mí, por más cursos que me des, no me vas a decir más si tengo que reciclar o no reciclar. Yo una vez que sé, y me conciencio de que tengo que reciclar, yo no necesito más cursos. Ahora, quizás otra gente necesite también cursos, eso sí es verdad, pero la misma gente más cursos no. Si está concienciado está concienciado. Lo que tienes que poner es medios y saber si los necesita o no, pero más concienciación...

Se observa que la palabra formación genera inquietud y desconfianza, como se puede observar en la cita anterior (fragmento 13): «Yo una vez que sé, y me conciencio de que tengo que reciclar, yo no necesito más cursos». De alguna forma, estos comerciantes rechazan recibir más cursos, o más concienciación, pero sí piden más medios materiales para poder actuar correctamente, o más información para los clientes.

En cambio, consideran que el intercambio de buenas prácticas podría serles más útil que una formación unidireccional dirigida solo a concienciarles, pero consideran que este tipo de aprendizaje de «buenas prácticas» no debería provenir del Ayuntamiento, sino de entidades que estén llevándolas a cabo o que tengan un conocimiento especial.

Fragmento 14. Tercera reunión de grupo

$\mathrm{H}$ : No es concienciarse sobre la necesidad o no de hacer estas cosas, sino formas para hacer cosas... Porque este tipo de cosas, el ahorrar algo de energía, por ejemplo, hay gente que lo está haciendo de mil maneras. Y esto, pues, se puede transmitir.

M: Mira, yo desde que vengo a esta charla por lo menos me he fijado en más cosas, he buscado más cosas.

H: Sí, estamos aquí, por eso digo...

Finalmente, sugieren que el Ayuntamiento debería apoyar más al pequeño comercio, dado que es indispensable para que haya actividad en la ciudad, para que se refuerce su vida social, algo que la propia A21L también comparte.

\section{Conclusiones}

Ante los temas ambientales y de sostenibilidad, existe una tendencia natural de la población a expresar discursos políticamente correctos, con el consiguiente riesgo de ocultación o disimulo de los ciudadanos a la hora de ser encuestados o entrevistados. Para paliar estas dificultades, el método STAVE pone en marcha un conjunto de mecanismos dirigidos a confrontar a las personas con sus propias opiniones y contradicciones discursivas, abriendo así una vía a la observación de sus prácticas cotidianas.

Los comerciantes de la muestra estudiada manifiestan una predisposición positiva hacia la sostenibilidad, tal como se ha podido observar a través de varios de los instrumentos utilizados en la aplicación del método STAVE (cuestionarios, grupos de discusión, diarios, etc.). Sin embargo, en su vida cotidiana, 
se encuentran con una serie de obstáculos que dificultan que aquellas actitudes o predisposiciones se puedan llevar a la práctica de manera conveniente. Sobre todo, enfatizan los obstáculos económicos, principalmente centrados en el consumo energético y en la gestión de residuos.

$\mathrm{Al}$ inicio del proceso de investigación, los comerciantes afirmaban tener una alta capacidad para avanzar hacia la sostenibilidad, pero, en sesiones posteriores, han ido reconociendo que se encuentran en una situación de dependencia respecto a otros actores (fabricantes, distribuidores, clientes), lo cual les impide adoptar decisiones efectivas para avanzar hacia prácticas más sostenibles. El reconocimiento de estos límites es fundamental para poder entender sus relaciones con la sostenibilidad y cómo les afectan las políticas ambientales existentes, lo cual puede ser de utilidad para su transformación o mejora.

Así, se observa que las soluciones que ofrece el Ayuntamiento a través de la A21L consisten, básicamente, en campañas informativas, formativas, de educación ambiental y de visibilización de buenas prácticas. No obstante, estas acciones, a pesar de su innegable interés y pertinencia, solo se aproximan tangencialmente a las necesidades de los comerciantes. De hecho, los comerciantes tienen la percepción de que el Ayuntamiento puede hacer pocas cosas. Desde su punto de vista, lo primero que se le exige es la provisión de recursos económicos (en forma de subvenciones, exenciones fiscales, etc.), aunque son conscientes de que los responsables de medio ambiente municipales no disponen precisamente de demasiados recursos, pero añaden también que se podría hacer «sensibilización» de los clientes, así como facilitar el acceso a ciertos objectos materiales a los comerciantes (bombillas de bajo consumo, bolsas de papel o biodegradables, etc.).

Se observa, también, que las iniciativas formativas que la A21L pone a disposición de los comerciantes no despiertan demasiado entusiasmo, pero ello no parece ser porque no necesiten formación, sino porque no les convence el formato (cursos, conferencias, etc.). Prefieren un aprendizaje basado en compartir experiencias con empresas que estén llevando a cabo iniciativas innovadoras en el ámbito de la sostenibilidad, donde ellos también puedan aportar sus conocimientos legos. Esto sí coincide con los postulados de la $\mathrm{A} 21 \mathrm{~L}$, que, mediante la red del programa Acción 21, procura generar interacciones entre empresas y entidades firmantes del Compromiso ciudadano por la sostenibilidad y en la promoción de buenas prácticas.

En definitiva, los obstáculos con que los comerciantes locales se encuentran en su vida cotidiana les dificultan poder cumplir con los objetivos del Compromiso ciudadano por la sostenibilidad impulsado por la A21L. La información generada mediante la aplicación del método STAVE puede ofrecer suficientes pistas a la A21L para diseñar un programa más adecuado para este colectivo, y las interacciones propiciadas entre la esfera ciudadana y la esfera de las personas que toman las decisiones políticas pueden ser un buen instrumento para introducir la lógica, las preocupaciones y las visiones ciudadanas en la elaboración de iniciativas ambientales a nivel local. 


\section{Agradecimientos}

Este artículo forma parte de los resultados del proyecto europeo PACHELBEL (Policy Addressing Climate Change and Learning about Consumer Behaviour and Everyday Life), financiado por la Comisión Europea (EU-FP7, grant agreement n. ${ }^{\circ}$ 244024). Agradecemos también la colaboración, buena predisposición y paciencia de las personas que formaban parte de la oficina técnica de la Agenda 21 de Barcelona y de las áreas de Medio Ambiente y de Participación del Ayuntamiento de Barcelona durante el periodo de estudio (2010-2012).

\section{Referencias bibliográficas}

Alaszewski, Andy (2006). Using Diaries for Social Research. Londres: Sage.

Ajuntament de Barcelona (2002a). Compromís ciutadà per la sostenibilitat. Barcelona: Ajuntament de Barcelona. Consell Municipal de Medi Ambient i Sostenibilitat.

- (2002b). Guí metodológica para avanzar hacia la sostenibilidad de Barcelona. Barcelona: Ajuntament de Barcelona. Consell Municipal de Medi Ambient i Sostenibilitat.

- (2011). 2a Convenció dels signants del "Compromis ciutadà per la sostenibilitat 20102011». Barcelona: Ajuntament de Barcelona. Consell Municipal de Medi Ambient i Sostenibilitat.

- (2012). Compromís ciutadà per la sostenibilitat 2012-2022: Per una Barcelona més pròspera, equitativa i autosuficient. Barcelona: Ajuntament de Barcelona. Consell Municipal de Medi Ambient i Sostenibilitat.

CG (Consell de Gremis) (2010). Agenda 21 i el comerç a Barcelona: Dades comparatives anys 2005-2010. Barcelona: Consell de Gremis de Comerç, Serveis i Turisme de Barcelona.

Espluga, Josep; Prades, Ana y Boso, Àlex (2011). «Com treballem?: Percepcions de l'Agenda 21 per part de les organitzacions signants del Compromís ciutadà per la sostenibilitat». En: Ajuntament de Barcelona. $2 a$ Convenció dels Signants del Compromis Ciutadà per la Sostenibilitat. Barcelona: Ajuntament de Barcelona. Consell Municipal de Medi Ambient i Sostenibilitat, 40-43.

- (2012). «Estratègies de comunicació del risc i modificació de pautes de comportament: Més enllà del model del dèficit». Periodística [en línea], 14, 25-43. $<$ http://dx.doi.org/10.2436/20.3008.02.23>.

Horlick-Jones, Tom (2005). «Informal logics of risk: Contingency and modes of practical reasoning». Journal of Risk Research [en línea], 8 (3), 253-272. <http://dx.doi.org/10.1080/1366987042000270735>.

- (2008). «Risk, praxis and everyday life». Comunicación presentada en el First ISA Congress. Barcelona, del 5 al 8 de septiembre.

Horlick-Jones, T. y Prades, A. (2014). «Translating between social worlds of policy and everyday life: The development of a group-based method to support policy-making by exploring behavioural aspects of sustainable consumption». Public Understanding of Science [en línea], abril. $<$ http://dx.doi.org/10.1177/0963662514525556>.

Horlick-Jones, Tom y RosenheAD, Jonathan (2002). «Investigating risk, organisations and decision support through action research». Risk Management [en línea], 4 (4), 45-63. $<$ http://dx.doi.org/10.1057/palgrave.rm.8240132>. 
- (2007). "The uses of observation: Combining problem structuring methods and ethnography». Journal of Operational Research Society [en línea], 58, 588-601. $<$ http://dx.doi.org/10.1057/palgrave.jors.2602271>.

Horlick-Jones, Tom; Walls, John y Kitzinger, Jenny (2007). «Bricolage in action: Learning about, making sense of, and discussing issues about genetically modified crops and food». Health, Risk \& Society [en línea], 9 (1), 83-103. <http://dx.doi.org/10.1080/13698570601181623>.

Prades, Ana; Holick-Jones, Tom; Barnett, Julie; Constantin, Marian; Enander, Ann; Espluga, Josep; Konrad, Wilfried; Poumadère, Marc y Rosenhead, Jonathan (2015). «PACHELBEL/STAVE: Shining a light on citizens' everyday environment-related behaviours». En: Martinuzzi, A. y Sedlacko, M. (eds.). Knowledge Brokerage for a Sustainable Europe: Innovative tools for enhancing the connectivity of research and policy making on sustainable development issues. Sheffield (UK): Greenleaf.

Rosenhead, Jonathan y Mingers, John (eds.) (2001). Rational Analysis for a Problematic World Revisited: Problem structured methods for complexity, uncertainty and conflict [en línea]. Chichester (UK): Wiley. <http://dx.doi.org/10.1002/sres.491>.

SANCASSIANI, Walter (2005). «Local agenda 21 in Italy: An effective governance tool for facilitating local communities' participation and promoting capacity building for sustainability». Local Environment: The International Journal of Justice and Sustainability [en línea], 10 (2), 189-200.

<http://dx.doi.org/10.1080/1354983052000330770>.

Zimmerman, D. H. y Wieder, D. L. (1977). "The diary interview method». Urban Life [en línea], 4 (5), 479-499.

$<$ http://dx.doi.org/10.1177/089124167700500406>. 